Research Article

\title{
Compound Fault Diagnosis of Rolling Bearing Based on ALIF-KELM
}

\author{
Jie Ma $(\mathbb{D}$, Shitong Liang $(\mathbb{D}$, Zhengyu Du, and Ming Chen \\ School of Mechatronics Engineering, Beijing Information Science and Technology University, Beijing, China \\ Correspondence should be addressed to Jie Ma; mjbeijing@163.com
}

Received 19 August 2021; Revised 25 September 2021; Accepted 12 October 2021; Published 26 October 2021

Academic Editor: Yong Chen

Copyright $\odot 2021$ Jie Ma et al. This is an open access article distributed under the Creative Commons Attribution License, which permits unrestricted use, distribution, and reproduction in any medium, provided the original work is properly cited.

\begin{abstract}
Aiming at the shortcomings of difficult classification of rolling bearing compound faults and low recognition accuracy, a composite fault diagnosis method of rolling bearing combined with ALIF and KELM is proposed. First, the basic concepts of ALIF and KELM are introduced, and then ALIF is used to decompose the sample data of vibration signals of different bearing states so that each sample can get several IMFs, select the top K IMFs containing the main fault information from each sample, calculate the energy feature and sample entropy of each IMF, and construct a fault feature vector with a dimension of $2 \mathrm{~K}$. Finally, the feature vectors of the training set and the test set are input into the KELM model for fault classification. Experimental results show that, compared with EMD-KELM model, ALIF-ELM model, ALIF-BP model, and IFD-KELM model, the rolling bearing composite fault diagnosis method based on the ALIF-KELM model has higher classification accuracy.
\end{abstract}

\section{Introduction}

Rolling bearings are one of the basic components and play an important role in various types of industrial equipment. Rolling bearings have been widely used in many engineering fields. However, the actual working environments of rolling bearings are very harsh. After an extended period of operation, these components are prone to failure. In addition to a single failure, the failure types can also easily present as composite failure formed due to simultaneous occurrences of multiple types of failures [1]. Statistical analysis [2] indicates that approximately $30 \%$ of all rotating machinery equipment failures are caused by failure of rolling bearings. Consequently, effective monitoring of the integrity health status of rolling bearings and timely elimination of hidden issues play an important role in ensuring safe and reliable equipment operation, reduction in economic and capital losses, and avoiding accidents.

In view of the above situation, most of the methods currently proposed by researchers are based on vibration signal processing composite fault diagnosis technology for rolling bearings, in which the signal decomposition method is one of the effective methods for processing vibration signals. In 1998, Huang et al. [3, 4] proposed an empirical mode decomposition (EMD) algorithm. Ma Xinna and others combined EMD with an adaptive notch filter to realize the adaptive separation and diagnosis of rolling bearing composite faults. However, due to the lack of EMD's strict mathematical theoretical derivation, singular points in the signal easily lead to modal aliasing occurrences. Cubic spline interpolation has either underfitting or overfitting and is unstable under the noise interferences. To effectively resolves rolling bearing problems, researchers have proposed many adaptive mode decomposition methods inspired by the idea of EMD, including local mean decomposition (LMD), empirical wavelet transform (EWT), and variational modal decomposition (VMD) [5-7]. Huang et al. [8] extended the local mean decomposition to a complex local mean decomposition and were successful in applying it to the composite fault diagnosis of rolling bearings. Zhu et al. [9] proposed a parameterized local eigenscale decomposition method for the discontinuity of the first derivative of the local eigenscale decomposition method, applied it to the composite fault simulation signal and the bearing experimental signal, and verified the method's performance. Effectiveness and superiority of the latter method are 
demonstrated by a comparative analysis. $\mathrm{Hu}$ et al. [10] optimized several important parameters in the variational modal decomposition to improve the decomposition performances. At the same time, they also used the 1.5-dimensional spectrum to suppress noise and enhance the impact signal, combining the two to achieve effective separation of composite faults in the rolling bearings. In order to improve the stability and convergence of the mean function of the upper and lower envelopes under disturbances, Lin et al. [11] proposed an iterative filter (IF) algorithm, which follows the same algorithm framework as EMD and uses low-pass filtering to obtain the upper and lower mean functions of the signal envelope. In 2016, Cicone et al. [12] used the basic solution system of Fokker-Planck (FP) differential equations as the filter function to extend the IF algorithm; they proposed the Adaptive Local Iterative Filter (ALIF) algorithm. ALIF can effectively analyse and process nonlinear and nonstationary signals. At present, the algorithm has been increasingly applied to the field of rotating machinery fault diagnosis, Chen et al. [13] combined ALIF and energy operator demodulation methods to effectively diagnose the fault characteristic frequencies of rolling bearings. Zhang et al. [14] proposed a method based on ALIF and high-order energy operator demodulation and successfully identified weak fault components during the early faults stages of rolling bearings; compared with the low-order energy operator demodulation method, this approach proves to be a superior method.

In recent years, machine learning technology has allowed for better success, through applying intelligent fault diagnosis algorithms [15-18]. Globally, scholars have continued to research intelligent recognition algorithms based on the BP neural network; these applications have been widely applied to the rolling bearing fault diagnosis achieving relatively sound academic resolutions [19-21]. The BP neural network requires performing iterative calculations during the learning process; sometimes, it falls into a local minimum, causing the algorithm to become time intensive, and the generalization ability of the network is very limited [22].

To address the above problems, Huang et al. [23] proposed an extreme learning machine (ELM) based on the single-hidden layer feedforward network (SLFN). The algorithm relies on its own performance and has gradually attracted the attention of scholars in diverse fields, including significance for the development of intelligent diagnosis technology for rolling bearing faults. For the nonstationary characteristics of bearing vibration signals, scholars, locally and abroad, usually use various nonstationary signal processing and analysis methods combined with the ELM algorithm to conduct intelligent diagnosis research on rolling bearing faults. $\mathrm{Xu}$ and $\mathrm{Ma}$ [24] used a combination of empirical wavelet transform and ELM to apply to the study of intelligent diagnosis of rolling bearing faults and provided bearing experimental data to prove the feasibility of this method. When the intelligent diagnosis model remains unchanged, the construction of the fault feature vector will have an important influence on the diagnosis effect of the intelligent fault diagnosis. KELM is an improved algorithm proposed by Huang et al. $[25,26]$ and is based on ELM. First, the original algorithm is optimized, and then the kernel function is used to replace the activation function of the hidden layer to make the model stable and universal. The KELM algorithm has improved generalization ability and is more suitable for solving multiclassification problems. This paper proposes a composite fault diagnosis method for rolling bearings that combines both Adaptive Local Iterative Filter (ALIF) and KELM approaches.

\section{Adaptive Local Iterative Filter Algorithm}

Adaptive Local Iterative Filter (ALIF) is a new type of adaptive mode decomposition method; improvements are due to the iterative filtering algorithm (IF). ALIF mainly constructs a filter function with adaptive characteristics by applying the basic solution system of Fokker-Planck differential equations. Therefore, it is also very necessary to introduce the IF algorithm before introducing the principle of the ALIF algorithm.

2.1. Iterative Filter. IF is similar to the EMD algorithm; it iteratively filters out each eigenmode function (IMF) component. This method convolves the filter functions with the signal to be decomposed to obtain the sliding operator; this process replaces the process of fitting the original data to obtain the mean value of the envelope in the EMD algorithm. IF mainly includes two processes: inner loop and outer loop.

Knowing the preprocessed signal $X(t)$ and the filter function $f(t)$, the sliding operator $\Gamma(X(t))$ is obtained by calculating the convolution of $X(t)$ and $f(t)$ :

$$
\Gamma(X(t))=\int_{-h(z)}^{h(z)} X(t+\tau) f(t) \mathrm{d} \tau,
$$

where $f(t)$ is the fixed low-pass filter function; $h(z)$ is the filter interval; its calculation expression is as follows:

$$
h(z)=2\left[\frac{N \lambda}{a}\right]
$$

where $N$ is the signal length of $X(t) ; \lambda$ is the set value; $a$ is the number of extreme points of $X(t)$.

Then calculate the fluctuation operator $K(X(t))$ by preprocessing the difference between the signal $X(t)$ and the sliding operator $\Gamma(X(t))$ :

$$
K(X(t))=X(t)-\Gamma(X(t)) .
$$

Finally, it judges whether the volatility operator $K(X(t))$ meets the conditions of the IMF component, and only the volatility operator that meets the set conditions can be extracted as the IMF component. If not, the volatility operator needs to be screened further, and the specific process is as follows:

(1) Calculate the filtering interval 1 of the preprocessed signal according to formula (2). 
(2) Solve the sliding operator $\Gamma(X(t))$ according to formula (1).

(3) Calculate the volatility operator $K(X(t))$ according to formula (3), and the expression of the volatility operator in the screening process is as follows:

$$
\mathrm{K}_{n}(X(t))=X_{n}(t)-\Gamma_{n}(X(t))=X_{(n+1)}(t) .
$$

Let

$$
\operatorname{IMF}(t)=\lim _{n \longrightarrow \infty} \mathrm{K}_{n}(X(t)) .
$$

When IMF $(t)$ can meet the IMF component conditions, complete the extraction of IMF components; otherwise, continue to repeat steps 1 to 4 until the conditions are met before stopping the screening. However, in actual situations, it is impossible for $n$ to approach infinity, so the screening termination conditions for IMF components can be artificially set as follows:

$$
\sigma=\frac{\left\|K_{i, n}-K_{i, n-1}\right\|_{2}}{\left\|K_{i, n-1}\right\|_{2}}
$$

That is, when $\sigma$ is less than a specified threshold, the screening is stopped and $\operatorname{IMF}(t)$ is the filtered IMF component.

The above-mentioned is the inner circulation process, and its main purpose is to extract the qualified IMF components, while the function of the outer circulation process is to stop the inner circulation process. First, the margin after all the effective IMF components of the preprocessed signal are successfully extracted is defined as the residual signal, denoted as $c(t)$ :

$$
c(t)=X(t)-\operatorname{IMF}(t) .
$$

When the residual signal $c(t)$ has obvious trend characteristics, that is, there is only one extreme point at most, the entire iterative filtering process is halted. Otherwise, it needs to be used as a fresh preprocessing signal to continue to extract qualified IMF components.

2.2. Adaptive Local Iterative Filter. In IF, in order to reduce the negative impact of noise on it, filter functions are generally set in advance, but some complex signals will lack adaptability when applying IF algorithms and may also cause component waveform distortion. In order to analyse both nonlinear and nonstationary signals more effectively and overcome the shortcomings of the IF algorithm, Cicone et al. were inspired by the diffusion process of partial differential equations and used the solution of the Fokker-Planck equation to construct a filter function; this enabled the filter to be tightly supported in the time domain; its length can be flexibly changed and adaptability is enhanced. Moreover, it can also avoid false components in the iterative filtering process [14]. This allows ALIF to effectively suppress noise sensitivity and modal aliasing in the IF algorithm.
For interval $(a, b)$, there are two differentiable functions $p(x)$ and $q(x)$, and the following two conditions are satisfied:

(1) $p(a)=p(b)=0$, and $p(x)>0$ holds for $x \in(a, b)$

(2) $q(a)<0<q(b)$

The Fokker-Planck equation is as follows:

$$
\begin{aligned}
\frac{\partial}{\partial t} g(x, t)= & -\alpha \frac{\partial}{\partial x}[p(x, t) g(x, t)]+\beta \frac{\partial^{2}}{\partial x^{2}} \\
& \cdot\left[q^{2}(x, t) g(x, t)\right], \quad \alpha, \beta>0 .
\end{aligned}
$$

In order to simplify the above formula, convert to the expression of the differential equation:

$$
G_{t}=-\alpha(p(x) g)_{x}+\beta\left(q^{2}(x) g\right)_{x x},
$$

where $\alpha$ and $\beta$ are called steady-state coefficients and $\alpha, \beta \in(0,1)$.

$(p(x) g)_{x}$ in equation (9) will have the effect of aggregation so that the solution $g(x)$ of the equation will start from the two endpoints of the interval $[a, b]$ towards the center point. At the same time, $\left(q^{2}(x) g\right)_{x x}$ will produce the effect of diffusion, which causes the solution $g(x)$ of the equation to diffusely move from the center of interval $[a, b]$ to the two endpoints. When the two effects are balanced,

$$
-\alpha(p(x) g)_{x}+\beta\left(q^{2}(x) g\right)_{x x}=0 .
$$

At this time, the differential equation has a nonzero solution and meets the following conditions:

$$
\begin{array}{ll}
\forall x \in(a, b), & g(x)>0, \\
\forall x \notin(a, b), & g(x)=0 .
\end{array}
$$

The solution $g(x)$ in the Fokker-Planck equation is the filter function $f(t)$ used in iterative filtering. For different intervals $[a, b]$, the solution obtained by the filter function $f(t)$ will also be different, and the function expression will also differ, allowing the ALIF algorithm to solve for filter function adaptively.

\section{Kernel-Based Extreme Learning Machine Algorithm}

3.1. Extreme Learning Machine. The ELM network structure is shown in Figure 1. The network structure includes three layers: hidden layer, input layer, and output layer [27]. The ELM intelligent learning model needs to provide the number of hidden layer nodes and the type of activation function during the entire learning process, while the input weights and hidden layer thresholds are randomly generated and remain unchanged. Finally, the least square method can be used to solve the output weight under the premise of ensuring that the training error is minimized.

Assuming that there are $N$ existing data samples $\left(x_{i}, t_{i}\right)$ that are not specific, where $x_{i}=\left[x_{i 1}, x_{i 2}, \ldots, x_{i n}\right]^{T} \in R^{N}$ and $t_{i}=\left[t_{i 1}, t_{i 2}, \ldots, t_{i n}\right]^{T} \in R^{N}$, the corresponding output expression of SLFN with $L$ hidden layer nodes is as follows: 


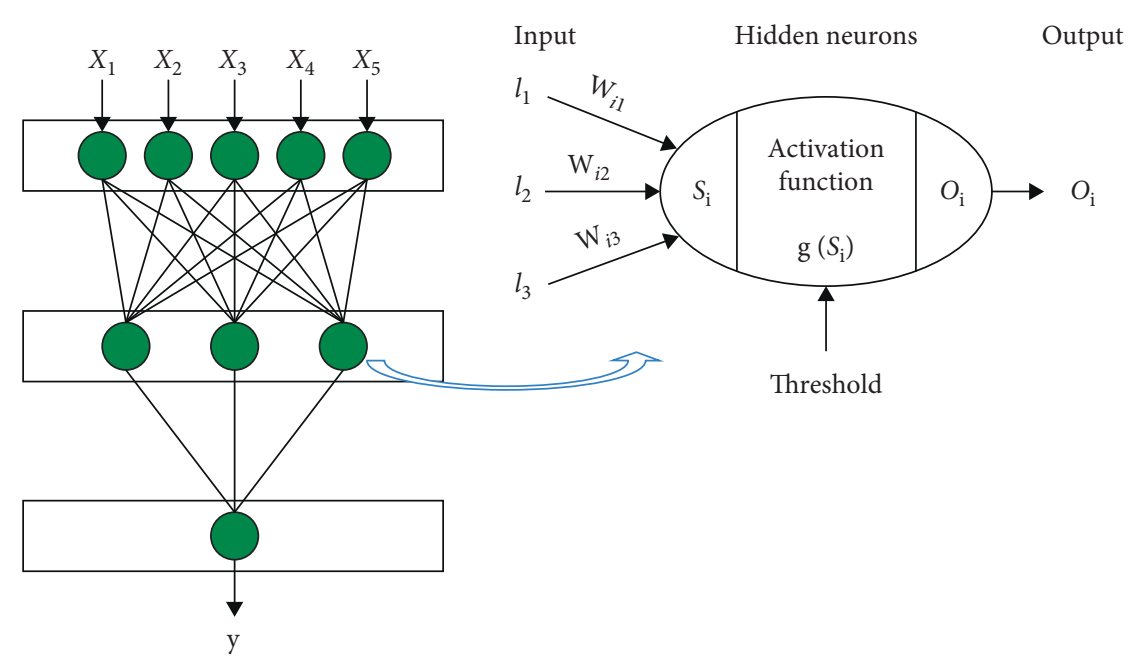

FIGURE 1: ELM network structure diagram.

$$
y=\sum_{i=1}^{L} \beta_{i} g\left(\omega_{i} \cdot x_{j}+b_{i}\right)=t_{j}, \quad j=1,2, \ldots, N,
$$

where $\beta_{i}$ represents the connection weight of the output layer and the hidden layer; $\omega_{i}$ represents the connection weight of the input layer and the hidden layer; $x_{j}$ is the input vector, which also represents all the feature vectors of the $j$ th sample; $b_{i}$ represents the hidden layer threshold; $g(x)$ represents the hidden layer containing layer activation function; $t_{j}$ is the output vector, which also represents the class label of the $j$ th sample.

Assuming that the activation function $g(x)$ is infinitely differentiable, then the ultimate goal of ELM learning is to minimize the output error; that is, infinity approaches 0 , which can be expressed as follows:

$$
\sum_{j=1}^{N} t_{j}-T_{j}=0
$$

Then there are $\beta_{i}$, $\omega_{i}$, and $b_{i}$ that make the following formula true:

$$
\sum_{i=1}^{L} \beta_{i} g\left(\omega_{i} \cdot x_{j}+b_{i}\right)-T_{j}=0, \quad j=1,2, \ldots, N .
$$

The abbreviated formula (14) is expressed as a matrix form as follows:

$$
H \beta=T,
$$

where $H$ is the hidden layer output matrix; $T$ is the expected output matrix; they are represented as follows:

$$
\begin{aligned}
H(\omega, x, b) & =\left[\begin{array}{ccc}
g\left(\omega_{1} \cdot x_{1}+b_{1}\right) & \cdots & g\left(\omega_{L} \cdot x_{1}+b_{L}\right) \\
g\left(\omega_{1} \cdot x_{2}+b_{1}\right) & \cdots & g\left(\omega_{L} \cdot x_{2}+b_{L}\right) \\
\vdots & \ddots & \vdots \\
g\left(\omega_{1} \cdot x_{N}+b_{1}\right) & \cdots & g\left(\omega_{L} \cdot x_{N}+b_{L}\right)
\end{array}\right]_{N \times L}, \\
\beta & =\left[\beta_{1}, \beta_{2}, \ldots, \beta_{L}\right]_{m \times L}^{T}, \\
T & =\left[t_{1}, t_{2}, \ldots, t_{N}\right]_{m \times N}^{T} .
\end{aligned}
$$

Since the input parameters of the ELM algorithm are randomly generated and remain unchanged, there is no need to adjust during the entire training and learning process. The connection weight 1 of the output layer and the hidden layer under the minimum error can be solved by the following formula:

$$
\widehat{\beta}=H^{+} T
$$

where $\mathrm{H}^{+}$represents the Moore-Penrose generalized inverse matrix of $H$.

3.2. Kernel-Based Extreme Learning Machine. The kernel extreme learning machine is based on the single-hidden layer feedforward neural network extreme learning machine. By introducing the kernel function mapping and regularization theory to optimize the model network, it can improve the accuracy and generalization ability while reducing the complexity and randomness of the network.

The extreme learning machine can be expressed by the following formula through mathematical expression:

$$
\begin{array}{ll}
\min : & L_{p}=\frac{1}{2} \beta_{i}^{2}+\frac{C}{2} \sum_{i=1}^{N} \xi_{i}^{2}, \\
\text { s.t.: } \quad h\left(x_{i}\right) \beta_{i}=t_{i}-\xi_{i}, \quad i=1,2, \ldots, N .
\end{array}
$$

where $C$ represents the penalty coefficient; $\xi$ is the training error; $h(x)$ is the output row vector of the hidden layer.

Solving for the above optimization problem, it can be concluded that the improved output function of ELM is as follows:

$$
f\left(x_{i}\right)=h\left(x_{i}\right) H^{T}\left(\frac{1}{C}+H H^{T}\right)^{-1} T,
$$

where $H$ is expressed as follows:

$$
H=\left[h\left(x_{1}\right), \ldots, h\left(x_{N}\right)\right]_{N \times L}^{T} .
$$


Regarding $h\left(x_{i}\right)$ as the nonlinear mapping of each sample, $H H^{T}$ represents the inner product form of $h\left(x_{i}\right)$, using the kernel function theory to define the kernel matrix $\Omega_{\mathrm{ELM}}$ to replace $H H^{T}$ so as to overcome the fluctuation of the final result of the ELM algorithm due to randomly generated inputs. The kernel matrix definition of KELM is as follows:

$$
\begin{aligned}
\Omega_{\mathrm{ELM}} & =H H^{T}, \\
\Omega_{\mathrm{ELM}_{i j}} & =h\left(x_{i}\right) \cdot h\left(x_{j}\right)=K\left(x_{i}, x_{j}\right) .
\end{aligned}
$$

After finishing formulas (20) (22) and substituting them into formula (19), the new output function $e$ of KELM is obtained as follows:

$$
f(x)=\left[\begin{array}{c}
K\left(x, x_{1}\right) \\
\vdots \\
K\left(x, x_{N}\right)
\end{array}\right]\left(\frac{1}{C}+\Omega_{\mathrm{ELM}}\right)^{-1} T,
$$

where $T$ represents the label of the data set; $\Omega_{\mathrm{ELM}}$ is a symmetric matrix with $N$ rows and $N$ columns; $K\left(x_{i}, x_{j}\right)$ is the kernel function quoted. This paper uses the Gaussian radial basis kernel function; $\Omega_{\mathrm{ELM}}$ and $K\left(x_{i}, x_{j}\right)$ are expressed in the following specific forms:

$$
\begin{aligned}
\Omega_{\mathrm{ELM}} & =\left[\begin{array}{ccc}
K\left(x_{1}, x_{1}\right) & \cdots & K\left(x_{1}, x_{N}\right) \\
\vdots & \ddots & \vdots \\
K\left(x_{N}, x_{1}\right) & \cdots & K\left(x_{N}, x_{N}\right)
\end{array}\right]_{N \times N}, \\
K\left(x_{i}, x_{j}\right) & =\exp \left(-\lambda x_{i}-x_{j}^{2}\right), \quad \lambda>0 .
\end{aligned}
$$

where $\lambda$ represents the nuclear coefficient.

\section{Classification Algorithm Based on the Combination of ALIF and KELM}

According to the previous introduction and analysis, ALIF can effectively decompose nonlinear and nonstationary vibration signals and further analyse the IMF components obtained after decomposition. ALIF can extract the local characteristics of the fault signal. Compared to traditional neural network algorithms, KELM has a strong generalization learning ability and at the same time has high efficiency and stability. Therefore, this paper combines the two and proposes a diagnostic method for rolling bearing composite faults based on ALIF and KELM. The specific steps are as follows:

(1) The ALIF decomposition is applied to the sample data of the vibration signal of different bearing states, and each sample can get several IMF components and a residual component

(2) Select the first $K$ IMF components containing the main fault information from each sample, calculate the energy characteristics and sample entropy of each component, and fuse them to construct a fault feature vector with a dimension of $2 \mathrm{~K}$
(3) Divide all samples into training samples and test samples in a certain proportion

(4) Select the specific form of the kernel function, and determine the two parameters of the kernel coefficient $\lambda$ and the penalty coefficient $C$ to complete the initialization of the KELM intelligent diagnosis model

(5) The fault feature vector of each sample is normalized to improve the comparability between data

(6) Let the KELM intelligent diagnosis model continuously learn through the fault feature vector set of the training sample, then test the fault feature vector set of the test sample, and finally, identify different bearing fault types and output the results

The corresponding flowchart of the steps outlined is shown in Figure 2.

\section{Classification Experiment}

5.1. Experimental Data Processing. The data set analysis and verification in this study were generated from the Xi'an Jiaotong University rolling bearing accelerated life test. The experimental setup is shown in Figure 3 [28]. The sampling frequency is set to $25.6 \mathrm{kHz}$, the sampling interval is set to $1 \mathrm{~min}$, and each sampling time is $1.28 \mathrm{~s}$, so the number of sampling points for each sample in the data set is 32768 . The vibration signal collected in the experiment is all the data of the rolling bearing from normal to failure, including a total of 15 data sets under 3 working conditions. In the following sections, four data sets will be used to analyse and verify the method proposed in this paper. The data description is shown in Table 1. The bearing data used in the subsequent analysis in this paper are based on the failure data intercepted during the whole life cycle.

This paper uses the experimental data set introduced in Table 1 and obtains 102400 sample points of rolling bearing outer ring fault, cage fault, inner ring and outer ring composite fault, inner ring fault, and normal state data from it.

The data of each state of the rolling bearing is divided into 50 samples, a total of 250 samples are obtained from the five states, and each sample contains 2048 sampling points. The procedure is to take one sample from each of the five bearing states and generate their time-domain waveforms as shown in Figure 4.

5.2. Fault Feature Analysis. It is necessary to construct fault feature vectors in advance before KELM performs intelligent fault diagnosis. Selecting appropriate features will help improve the accuracy of fault intelligent diagnosis. Therefore, before proceeding with the method verification, a brief analysis of the fault characteristics used in this paper is given.

Select one sample data in each of the five states of the rolling bearing, set the same parameters for all five samples, and then apply ALIF decomposition to obtain five IMF components and one residual component. Calculate the energy characteristics and sample entropy of the first 4 IMF 


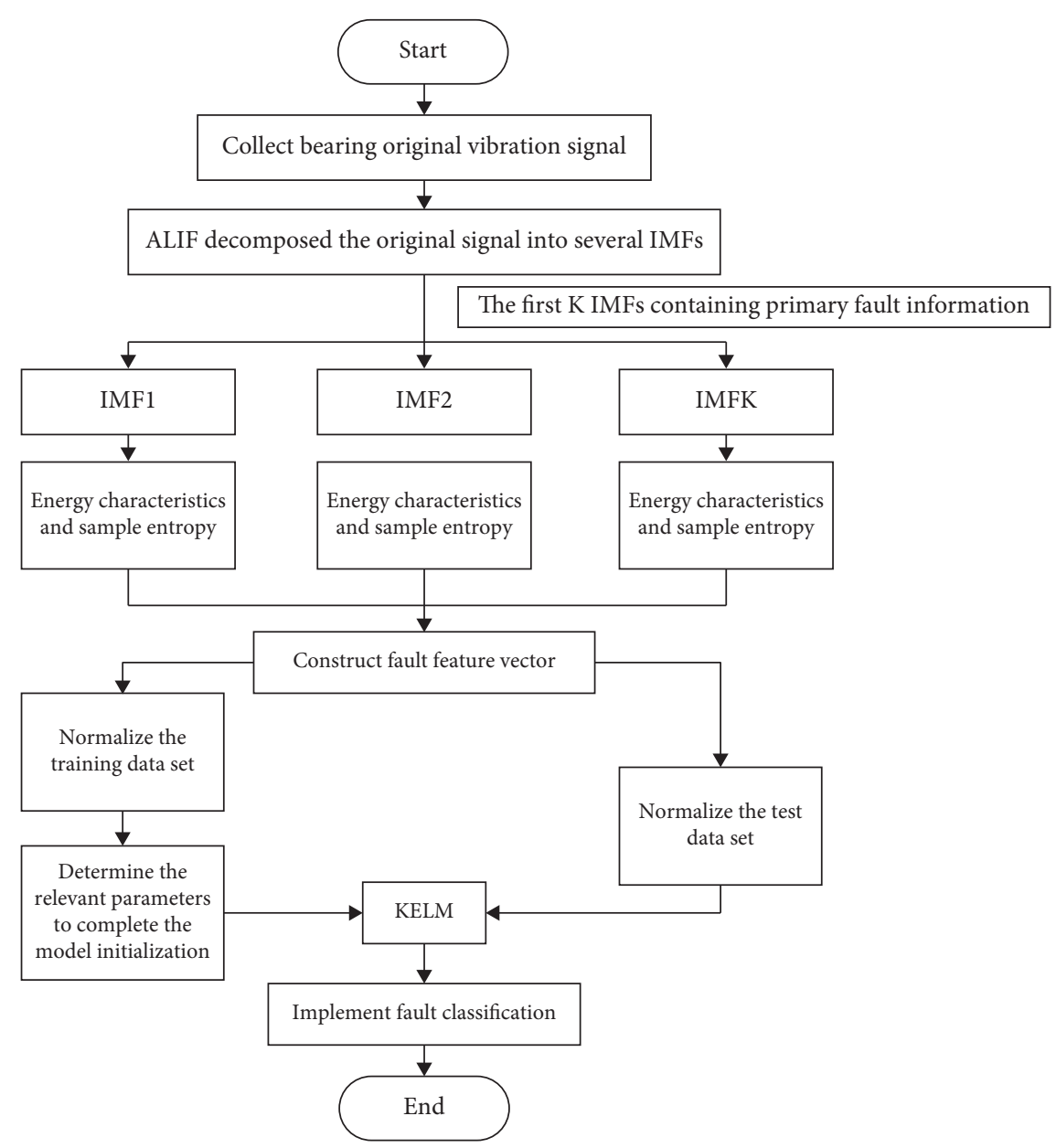

FIgURe 2: Fault diagnosis flowchart based on ALIF and KELM.

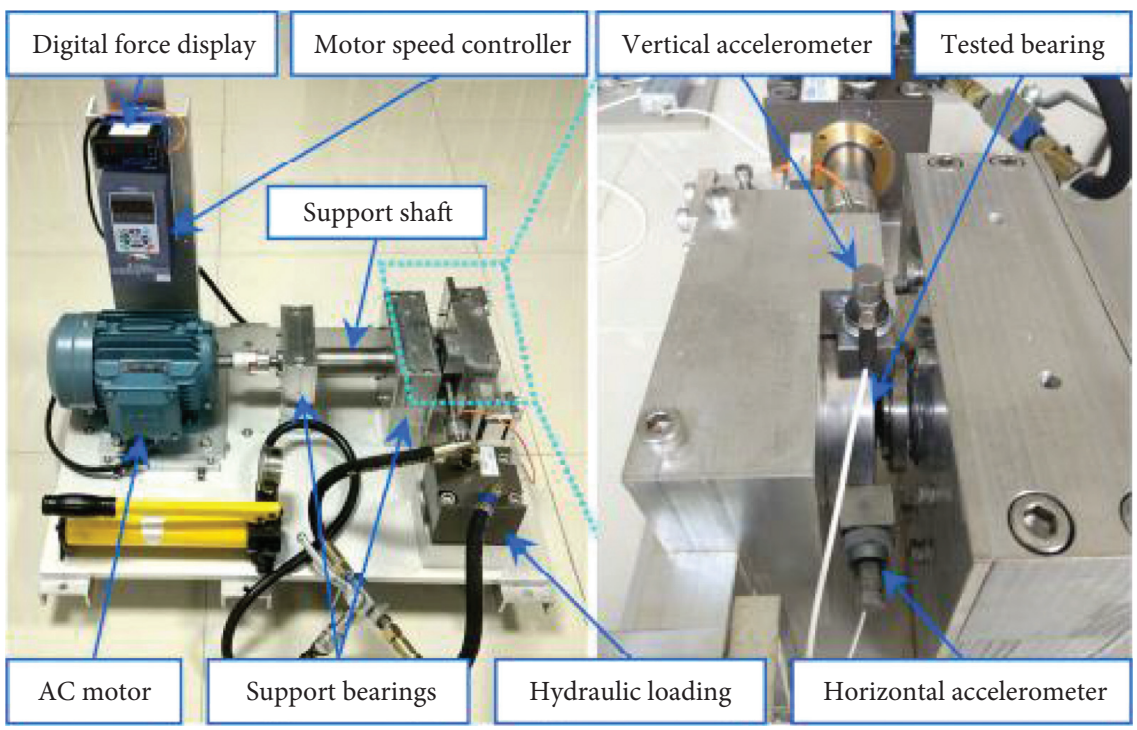

FIGURE 3: Rolling bearing accelerated life test bench.

components; the results are shown in Figures 5(a) and 5(b). It can be seen from both graphs that the energy characteristics of the first four IMF components and the sample entropy of the first four IMF components decomposed by
ALIF are different under different operating conditions. However, if only energy is selected as the feature vector, there are three kinds of bearing state features in the IMF1 component that have obvious aliasing, and there are also two 
TABLE 1: Dataset details.

\begin{tabular}{lcc}
\hline Dataset & Fault type & Rotating speed $(\mathrm{r} / \mathrm{min})$ \\
\hline Bearing1 & Outer ring failure & 2100 \\
Bearing2 & Compound failure of inner ring and outer ring & 2100 \\
Bearing3 & Inner ring failure & 2250 \\
Bearing4 & Cage failure & 2250
\end{tabular}
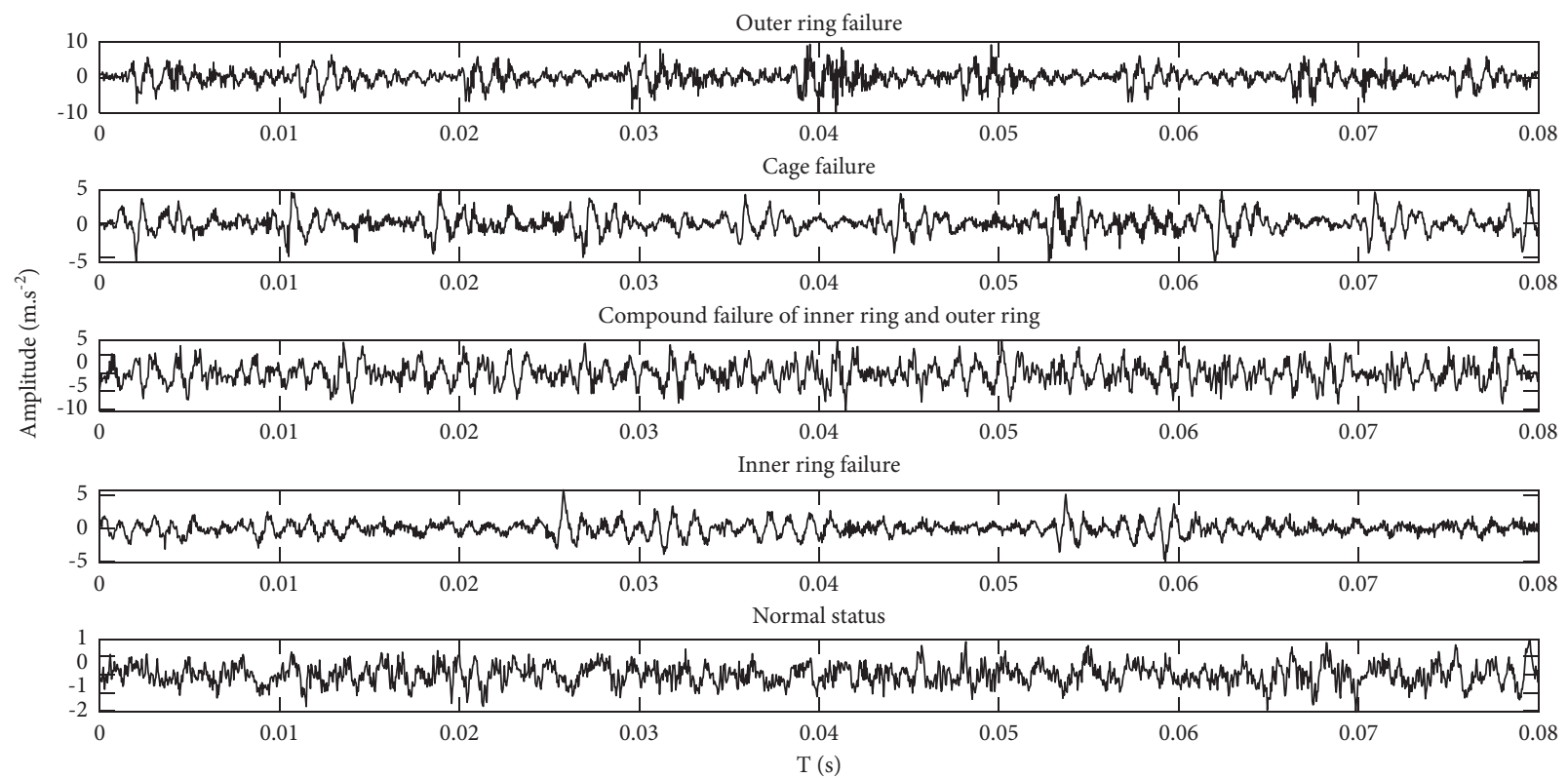

Figure 4: Time-domain waveforms of rolling bearing samples.

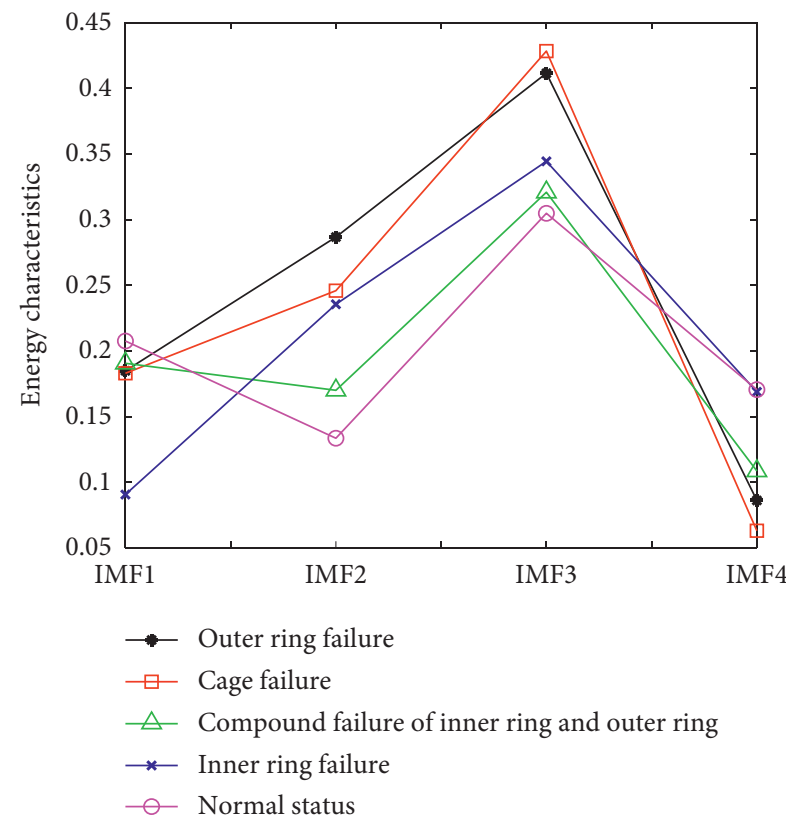

(a)

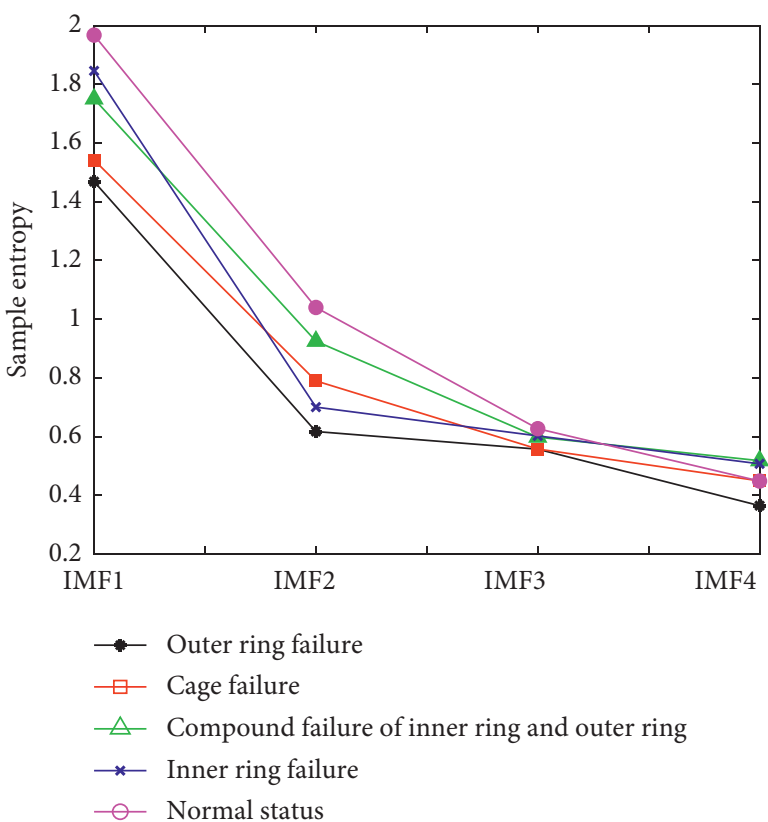

(b)

FiguRE 5: Energy characteristics and sample entropy of the first 4 components. (a) The energy characteristic value of the first 4 components.

(b) The value of the sample entropy of the first 4 components. 
bearing state features in the IMF4 component that basically overlap. This has been an obstacle to identifying types of bearing faults. When the energy feature and the sample entropy are selected as the fault feature vector, several bearing states where the original energy features are aliased can be effectively distinguished by the sample entropy.

5.3. Experimental Results and Analysis. In order to intuitively distinguish the different operating states of rolling bearings in the subsequent analysis, the sample data of the 4 types of faults and normal states are divided into 5 categories, and the specific category labels are given as shown in Table 2.

First, use the ALIF algorithm to decompose the vibration signals of all samples, make it get 5 IMF components and 1 residual component, calculate the energy characteristics and sample entropy of the first 4 IMF components, and obtain a fault eigenvector matrix with a size of $250 \times 8$. Then select 30 samples in each state as the training set and 20 samples as the test set, and normalize the fault feature data set to make the data indicators.

Finally, the Gaussian radial basis kernel function in equation (25) is used as the kernel function of KELM; the kernel coefficient $\lambda=0.5$ and penalty coefficient $C=1$ are determined. The initialization of the KELM intelligent diagnosis model is then completed. Input 150 training sample sets into the KELM model for training, and then apply 100 test samples for testing; the result is shown in Figure 6.

The abscissa in Figure 6 represents 100 sets of test samples, and each of the 5 bearing failure categories uses 20 sets of samples as the test; the vertical axis shows the category labels of different failures of rolling bearings, corresponding to Table 2. Judging from the diagnostic results, only one of the 100 test samples was misdiagnosed. The composite fault of the inner ring and the outer ring was misdiagnosed as a cage failure, while the remaining 99 test samples were accurately diagnosed. Taken together, the overall fault diagnosis accuracy rate is $99 \%$, of which the single fault diagnosis accuracy rate is $100 \%$, and the compound fault diagnosis accuracy rate is $95 \%$. Therefore, the effectiveness of the intelligent diagnosis method of rolling bearing composite fault based on ALIF and KELM proposed in the paper can be proved.

However, in practical engineering applications, the sample data available for equipment is usually limited. The next step will be to study whether the method proposed in this paper can achieve higher accuracy fault intelligent diagnosis on test samples with fewer training samples. Setting the number of training samples for each failure type to 30 , $25,20,15,10,5$, and 1 in turn, the number of corresponding test samples is $20,25,30,35,40,45$, and 49 ; then we can get 7 different results of ALIF-KELM's diagnosis of rolling bearing faults. The relationship between the number of different training samples and the diagnosis accuracy is shown in Figure 7. It can be found that even when the number of training samples is only 1 , the overall accuracy of ALIF-KELM can still reach $81.63 \%$. When the number of training samples is 10 , the fault intelligent diagnosis
TABLE 2: Rolling bearing failure type label.

\begin{tabular}{lc}
\hline Fault type & Label \\
\hline Outer ring failure & 1 \\
Cage failure & 2 \\
Compound failure of inner ring and outer ring & 3 \\
Inner ring failure & 4 \\
Normal status & 5 \\
\hline
\end{tabular}

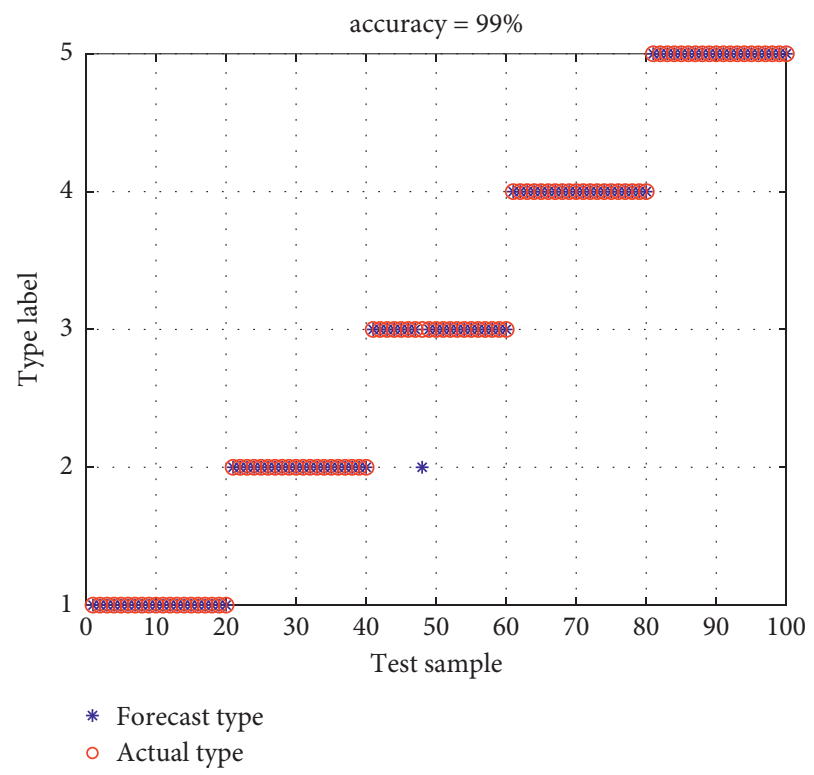

Figure 6: ALIF-KELM fault diagnosis results.

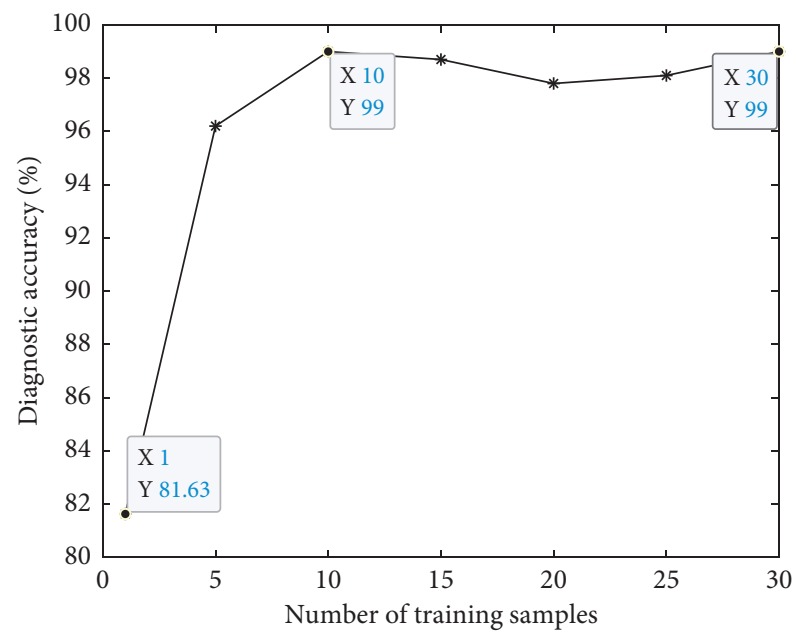

FIGURE 7: The fault diagnosis accuracy rate of ALIF-KELM under a different number of training samples.

accuracy can also be as high as $99 \%$; the specific diagnosis is shown in Figure 8. At this time, in all 200 test samples, only 2 sets of inner and outer ring compound faults were misdiagnosed as cage faults, so the compound fault accuracy rate is still $95 \%$, which is the same as the accuracy rate when the number of training samples is 30 . Compared with the 


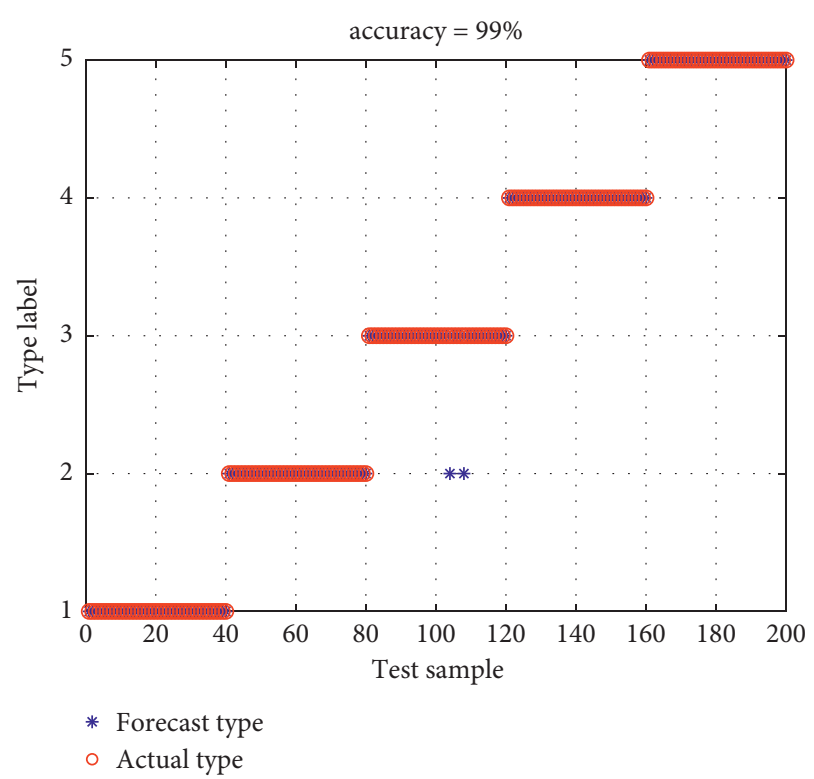

FIgURE 8: Fault diagnosis result of ALIF-KELM bearing under small sample.

IFD-KELM method proposed in [29], when the sample size is 10 , the accuracy of the method proposed in this paper is increased by $6.5 \%$. Therefore, it can be further proved that the intelligent diagnosis method of rolling bearing composite fault based on ALIF and KELM proposed in this chapter can still be effective with less sample data.

5.4. Comparative Analysis of Experiments. In order to verify that the energy characteristics and sample entropy of the first four IMF components calculated after the application of ALIF decomposition can more effectively reflect the different fault characteristics of rolling bearings, the ALIF decomposition method is replaced with the traditional decomposition method EMD for comparison. There are 10 training samples and 40 test samples in each bearing state, and the KELM parameters remain the same as before. A total of 50 training sample sets of five bearing states are input into the KELM model for training, and then 200 test samples are used for testing; the result is shown in Figure 9. The figure shows that there are a total of 5 groups of test samples with diagnostic errors. Among them, 3 groups misdiagnosed the outer ring fault as a cage fault and an inner ring fault, and 2 groups misdiagnosed the cage fault as a composite fault of the inner ring and the outer ring; the composite fault test samples are all accurately identified, but the overall fault diagnosis accuracy rate is $97.5 \%$. Figures 7 and 8 show that the overall diagnosis accuracy of ALIF-KELM is $99 \%$ in the case of 10 training samples. In contrast, the application of ALIF-KELM has higher accuracy than EMD-KELM's intelligent fault diagnosis method. It is verified that ALIF decomposition is more effective than EMD decomposition. In order to verify the superiority of the performance of the KELM fault diagnosis model, KELM was replaced with the two traditional diagnosis models of ELM and BP neural network. The method of feature extraction and the

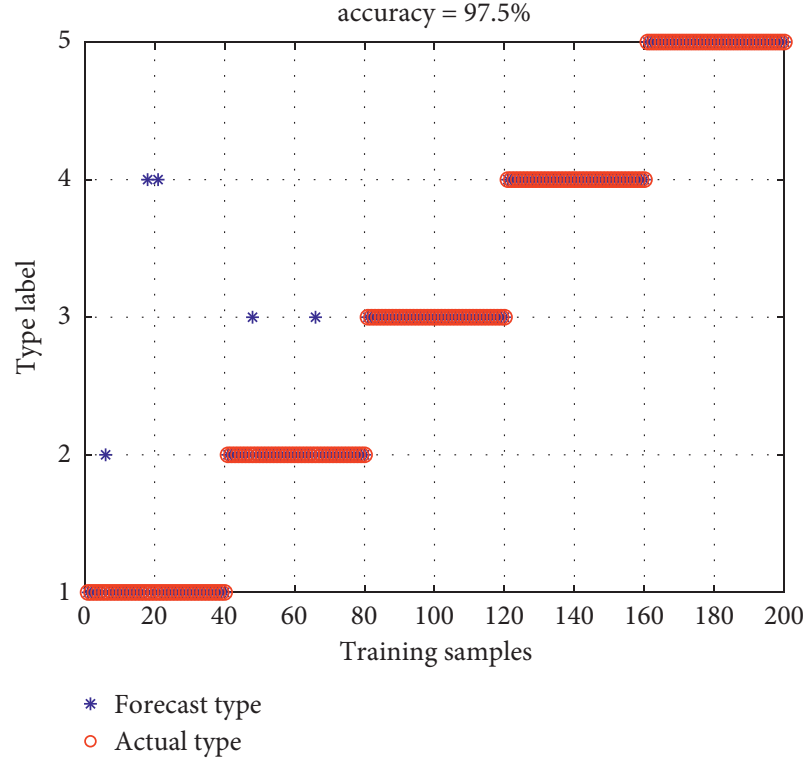

FIGURE 9: EMD-KELM bearing fault diagnosis results.

construction of feature vectors remain unchanged from the original method. The number of training samples in each bearing failure state is still 10, and the number of test samples is 40. Applying these two fault diagnosis models, respectively, the results are shown in Figures 10(a) and 10(b). Figure 10(a) shows that the accuracy of using ELM as a diagnostic model is $78.5 \%$. The accuracy of BP as a diagnostic model is lower; Figure 10(b) shows only 53\%. In short, compared with Figure 8, it can be found that the fault diagnosis accuracy of the two traditional diagnosis models is obviously much worse, and there are a lot of misdiagnosis phenomena in 200 test samples.

Since the initial weights of the two diagnostic models, ELM and BP, are randomly generated, the results obtained from each test are usually different. In order to reduce the impact of random fluctuations on the final comparison results, the three diagnostic models KELM, ELM, and BP were retrained and tested 10 times, and the accuracy of fault diagnosis was recorded. The results are shown in Figure 11. In order to be able to study the algorithm efficiency of each model and record the training time and test time consumed by each diagnostic model, the results are shown in Figures 12(a) and 12(b). Figure 10 shows that using KELM as an intelligent diagnosis model to identify fault types has not only the highest test accuracy but also the best stability. It can maintain an accuracy of $99 \%$ in 10 tests. Compared with the two diagnostic models of ELM and BP, the accuracy and stability of ELM are significantly better than that of BP. Figures 12(a) and 12(b) show that the KELM model is used for classification, the training time is the lowest, and the test time is basically the lowest, which is not much different from ELM, but the overall efficiency of KELM is the highest. And the algorithm efficiency of the BP model for classification is obviously lower than that of KELM and ELM. In short, ALIF-KELM has higher accuracy and algorithm efficiency than the two fault diagnosis methods ALIF-ELM and ALIF-BP. 


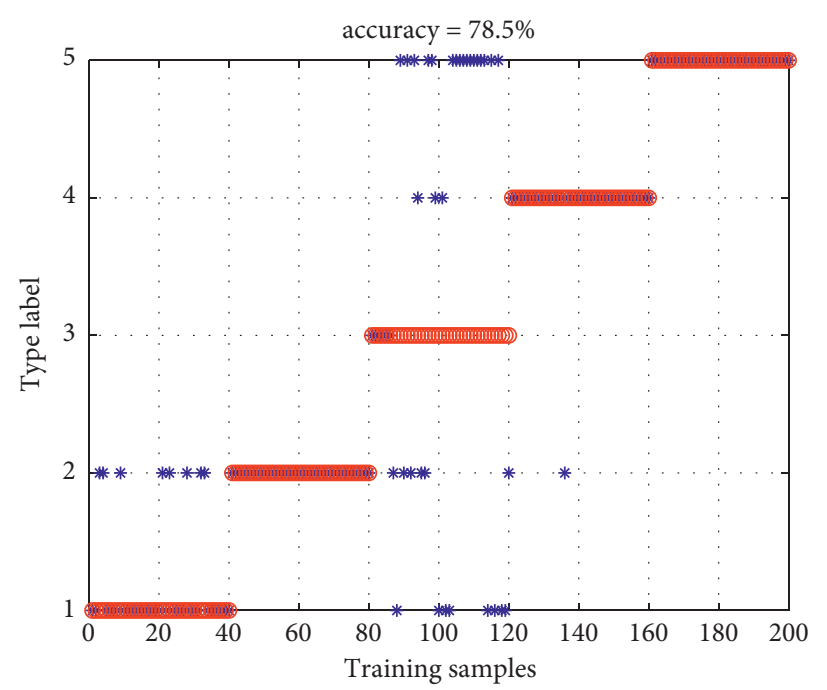

* Forecast type

- Actual type

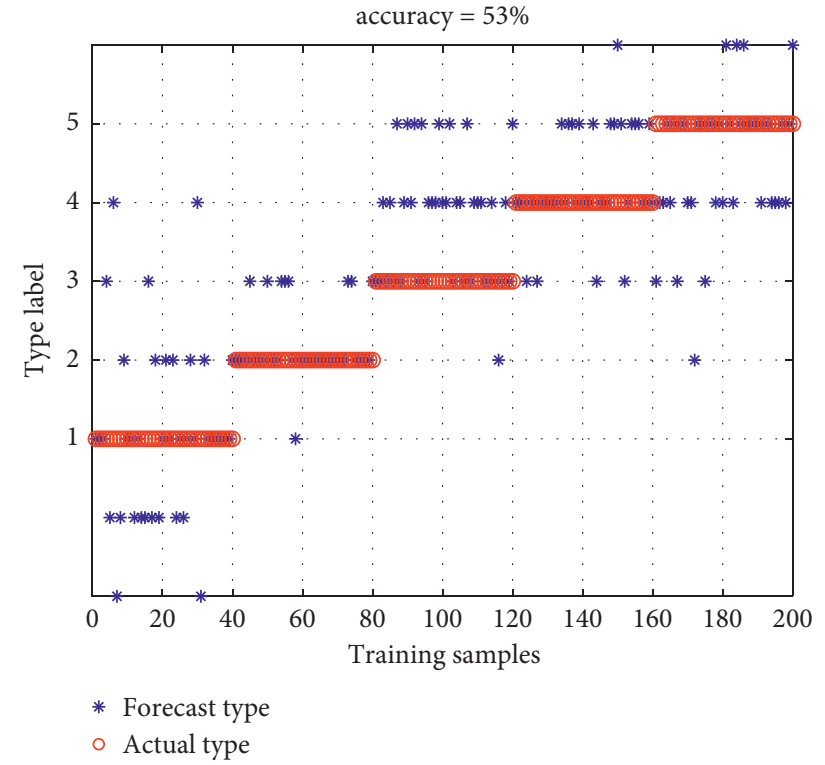

(b)

FIGURE 10: Diagnosis result of traditional diagnosis model. (a) ALIF-ELM fault diagnosis result. (b) EMD-BP fault diagnosis result.

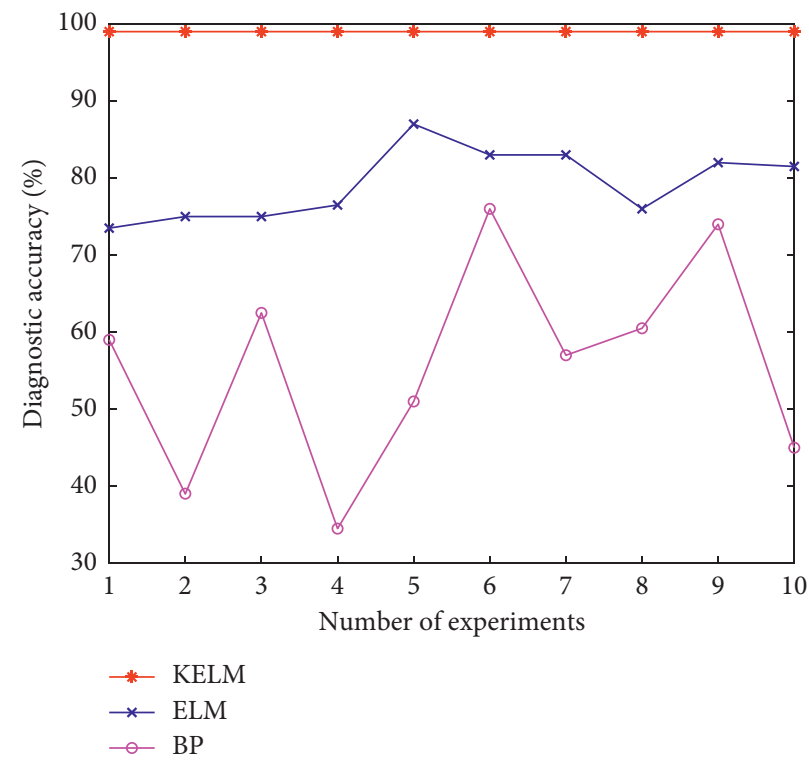

Figure 11: Accuracy of different fault diagnosis models. 


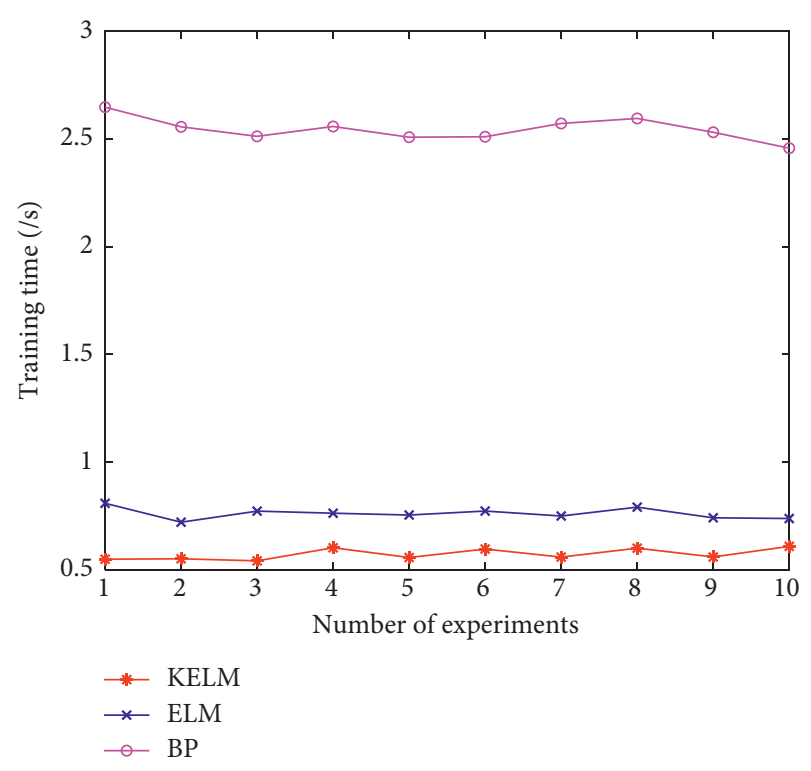

(a)

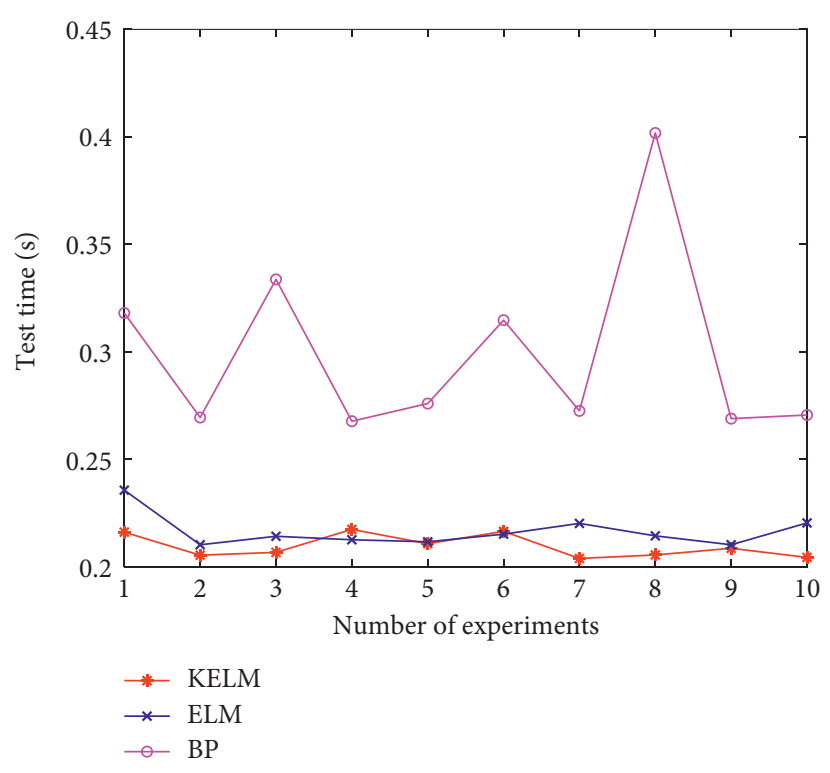

(b)

Figure 12: Time comparison of different fault diagnosis models. (a) Training time comparison. (b) Test time comparison.

\section{Conclusions}

In order to realize the composite fault diagnosis of rolling bearings, this paper proposes a diagnosis method by combining ALIF and KELM. In the case of a small sample, the proposed method is compared with EMD-KELM, ALIFELM, ALIF-BP, and IFD-KELM in [29]. The results show that both ALIF and KELM algorithms have certain advantages in the case of small samples, and the method proposed in this paper has high diagnostic accuracy and is suitable for the diagnosis of composite faults of rolling bearings.

\section{Data Availability}

The data used to support the findings of this study are available from the corresponding author upon request.

\section{Conflicts of Interest}

The authors declare that there are no conflicts of interest regarding the publication of this study.

\section{Acknowledgments}

This work was supported by the National Key Research and Development Program of China (Grant no. 2019YFB1705403) and the National Natural Science Foundation of China (Grant no. 61973041).

\section{References}

[1] K. Zhang, D. H. Zhou, and Y. Chai, "Review of multiple fault diagnosis methods," Control Theory \& Applications, vol. 32, no. 9, pp. 1143-1157, 2015.
[2] C. Li, M. Liang, and Z. Q. Chen, Intelligent Health Management of Rolling Bearings Based on Vibration Signal, Science Press, Beijing, China, 2018.

[3] N. E. Huang, Z. Shen, and S. R. Long, "The empirical mode decomposition and the Hilbert spectrum for nonlinear and non-stationary time series analysis," Proceedings of the Royal Society A: Mathematical, Physical \& Engineering Sciences, vol. 454, no. 1971, pp. 903-995, 1998.

[4] N. E. Huang, Z. Shen, and S. R. Long, "A new view of nonlinear water waves: the Hilbert spectrum," Annual Review of Fluid Mechanics, vol. 31, no. 1, pp. 417-457, 1999.

[5] Z. L. Liu, "Time-frequency representation based on robust local mean decomposition for multicomponent AM-FM signal analysis," Mechanical Systems and Signal Processing, vol. 95, pp. 468-487, 2017.

[6] Y. Li, D. Huang, and Z. Qin, "A classification algorithm of fault modes-integrated LSSVM and PSO with parameters' optimization of VMD," Mathematical Problems in Engineering, vol. 2021, Article ID 6627367, 12 pages, 2021.

[7] Z. Li, J. Chen, Y. Zi, and J. Pan, "Independence-oriented VMD to identify fault feature for wheel set bearing fault diagnosis of high speed locomotive," Mechanical Systems and Signal Processing, vol. 85, pp. 512-529, 2017.

[8] C. J. Huang, H. J. Song, and N. Qin, "Fault diagnosis method of rolling bearing based on CLMD and CSES," Electric Power Automation Equipment, vol. 40, no. 8, pp. 179-188, 2020.

[9] W. M. Zhu, Y. Yu, and H. F. Xue, "Parameterized local characteristic scale decomposition and its application in composite fault diagnosis," Journal of Mechanical \& Electrical Engineering, vol. 37, no. 6, pp. 593-599, 2020.

[10] A. J. Hu, Z. R. Bai, and J. Zhao, "Compound fault features separation method of rolling bearing based on parameter optimization VMD and 1.5 dimension spectrum," Journal of Vibration and Shock, vol. 39, no. 11, pp. 45-52+62, 2020.

[11] L. Lin, Y. Wang, and H. Zhou, "Iterative filtering as an alternative algorithm for empirical mode decomposition," Advances in Adaptive Analysis, vol. 1, no. 4, pp. 543-560, 2009. 
[12] A. Cicone, J. Liu, and H. Zhou, "Adaptive local iterative filtering for signal decomposition and instantaneous frequency analysis," Applied and Computational Harmonic Analysis, vol. 41, no. 2, pp. 384-411, 2016.

[13] B. J. Chen, X. B. Wang, and C. H. Zhao, "Fault feature extraction of rolling bearing based on ALIF and energy operator demodulation," Journal of Nanjing University of Science and Technology, vol. 42, no. 4, pp. 445-452, 2018.

[14] J. B. Zhang, Y. Q. Zhao, and M. Liu, "An improved higherorder analytical energy operator with adaptive local iterative filtering for early fault diagnosis of bearings," Journal of Vibroengineering, vol. 22, no. 1, 2020.

[15] J. Ma and J. Xu, "Fault prediction algorithm for multiple mode process based on reconstruction technique," Mathematical Problems in Engineering, vol. 2015, pp. 1-8, 2015.

[16] J. Viola, Y. Chen, and J. Wang, "FaultFace: deep convolutional generative adversarial network (DCGAN) based ball-bearing failure detection method," Information Sciences, vol. 542, pp. 195-211, 2021.

[17] J. Wang, W. Zhang, H. Wu, and J. Zhou, "Improved bilayer convolution transfer learning neural network for industrial fault detection," The Canadian Journal of Chemical Engineering, vol. 32, 2021.

[18] J. Wang, W. Zhang, and J. Zhou, "Fault detection with data imbalance conditions based on the improved bilayer convolutional neural network," Industrial \& Engineering Chemistry Research, vol. 59, no. 13, pp. 5891-5904, 2020.

[19] L. P. Huang, C. W. Wu, and J. Wang, "Fault pattern recognition of rolling using wavelet package analysis and BP neural network," Electronic Measurement Technology, vol. 39, no. 4, pp. 164-168, 2016.

[20] L. Huo, X. Y. Zhang, and H. D. Li, "Bearing Fault diagnosis based on BP neural network," IOP Conference Series: Earth and Environmental Science, vol. 208, no. 1, Article ID 012092, 2018.

[21] J. M. Li, X. F. Yao, and X. D. Wang, "Multiscale local features learning based on BP neural network for rolling bearing intelligent fault diagnosis," Measurement, vol. 153, Article ID 107419, 2020

[22] F. Han and D. S. Huang, "Improved extreme learning machine for function approximation by encoding a priori information," Neurocomputing, vol. 69, no. 16, 2006.

[23] G. B. Huang, Q. Y. Zhu, and C. K. Siew, "Extreme learning machine: a new learning scheme of feedforward neural networks," in Proceedings of the IEEE International Joint Conference on Neural Networks (IEEE Cat. No.04CH37541), pp. 985-990, Budapest, Hungary, July 2004.

[24] S. Xu and J. Ma, "Rolling bearing fault diagnosis based on EWT and ELM," Vibroengineering PROCEDIA, vol. 19, pp. $42-47,2018$.

[25] G.-B. Huang, "An insight into extreme learning machines: random neurons, random features and kernels," Cognitive Computation, vol. 6, no. 3, pp. 376-390, 2014.

[26] G. B. Guang-Bin Huang, H. Hongming Zhou, X. Xiaojian Ding, and fnm Rui Zhang, "Extreme learning machine for regression and multiclass classification," IEEE Transactions on Systems, Man, and Cybernetics, Part B (Cybernetics), vol. 42, no. 2, pp. 513-529, 2012.

[27] T. Liu, Research on Intelligent Optimization Based-Extreme Learning Machine with Application to Disease Diagnosis, Jilin University, Changchun, China, 2020.

[28] B. Wang, Y. Lei, N. Li, and N. Li, "A hybrid prognostics approach for estimating remaining useful life of rolling element bearings," IEEE Transactions on Reliability, vol. 69, no. 1, pp. 401-412, 2020.

[29] M. Q. Guo, Y. Y. Huang, and Q. Zhao, "Fault diagnosis method of rolling bearing based on IFD and KELM," Journal of Fuzhou University (Natural Science Edition), vol. 48, no. 3, pp. 341-347, 2020. 